\title{
ASYMMETRIC INNER PRODUCT AND THE ASYMMETRIC QUASI NORM FUNCTION
}

\author{
Stela Çeno ${ }^{1}$, Gladiola Tigno ${ }^{2}$
}

\begin{abstract}
This paper attempts to generalize the semi scalar product concept according to G. Lumer by replacing Cauchy inequality with another inequality which is more generalized. Based on this attempt of generalization it is built a function which fulfils the conditions which are changed. In this paper it is also generalized quasi norm function by replacing homogeneity condition with a more restricted condition by producing this time a more generalized asymmetric semi norm function. As a result, in this paper it is defined the asymmetric inner product function and the asymmetric quasi norm function. Moreover, it is even given relation between these two.
\end{abstract}

\section{INTRODUCTION}

Semi-inner products, that can be naturally defined in general Banach spaces over the real or complex number field, play an important role in describing the geometric properties of these spaces.

Starting from its axiomatic, many researchers have made various modifications passing in its generalization. Semi-scalar products mark the very first generalizations of the scalar product function. The strong bond between these functions with the norm function has made it possible to obtain a lot of interesting results which are connected with the orthogonality and convexity [1],[2].

In [3],[4] it is also generalized the quasi norm function by replacing homogeneity condition with a more restricted condition by producing this time a more generalized asymmetric semi norm function.

Let be $p_{0}: \mathbb{R} \rightarrow \mathbb{R}^{+}$a function defined by:

$$
p_{0}(x)=\left\{\begin{array}{l}
|x|, \quad x<0 \\
2|x|, x \geq 0
\end{array}\right. \text {. }
$$

2010 Mathematics Subject Classification. 46B20

Key words and phrases. Quasi norm function, asymmetric quasi inner product, asymmetric quasi norm function. 
Definition 1. The $p_{0}: \mathbb{R} \rightarrow \mathbb{R}^{+}$function is called an asymmetric semi norm if:

a) $p_{0}(x) \geq 0$ for $\forall x \in \mathbb{R}$.

b) $p_{0}(\lambda x)=\lambda p_{0}(x)$ for $\lambda>0, \forall x \in \mathbb{R}$

c) $p_{0}(x+y) \leq p_{0}(x)+p_{0}(y) \forall x, y \in \mathbb{R}$.

For every $x\left(x_{1}, x_{2}\right) \in \mathbb{R}^{2}$, we define the function $p(x)=p_{0}\left(x_{1}\right)+p_{0}\left(x_{2}\right)$, where $p_{0}\left(x_{1}\right), p_{0}\left(x_{2}\right)$ are asymmetric semi norms in $\mathbb{R}$.

Proposition 1. The function $p: \mathbb{R} \times \mathbb{R} \rightarrow \mathbb{R}$ such that $p(x)=p_{0}\left(x_{1}\right)+p_{0}\left(x_{2}\right)$ it is also an asymmetric semi norm in $\mathbb{R}^{2}$.

Proof. a) We have

b) We have

$$
\begin{aligned}
& p(x)=p_{0}\left(x_{1}\right)+p_{0}\left(x_{2}\right) \geq 0, \forall\left(x_{1}, x_{2}\right) \in \mathbb{R}^{2} \text { and } \\
& p(x)=0 \Rightarrow p_{0}\left(x_{1}\right)=0 \wedge p_{0}\left(x_{2}\right)=0 \Rightarrow x_{1}=x_{2}=0
\end{aligned}
$$

$$
\begin{aligned}
p(\lambda x) & =p_{0}\left(\lambda x_{1}\right)+p_{0}\left(\lambda x_{2}\right)=\lambda p_{0}\left(x_{1}\right)+\lambda p_{0}\left(x_{2}\right) \\
& =\lambda\left[p_{0}\left(x_{1}\right)+p_{0}\left(x_{2}\right)\right]=\lambda p(x), \text { for } \lambda>0
\end{aligned}
$$

c) We have

$$
\begin{aligned}
p(x+y) & =p_{0}\left(x_{1}+y_{1}\right)+p_{0}\left(x_{2}+y_{2}\right) \\
& \leq p_{0}\left(x_{1}\right)+p_{0}\left(y_{1}\right)+p_{0}\left(x_{2}\right)+p_{0}\left(y_{2}\right) \\
& =\left[p_{0}\left(x_{1}\right)+p_{0}\left(x_{2}\right)\right]+\left[p_{0}\left(y_{1}\right)+p_{0}\left(y_{2}\right)\right] \\
& =p(x)+p(y) .
\end{aligned}
$$

So $p(x+y) \leq p(x)+p(y), \forall x, y \in \mathbb{R}^{2}$.

For every two points $x\left(x_{1}, x_{2}\right)$ and $y\left(y_{1}, y_{2}\right)$ in $\mathbb{R}^{2}$ we build the function $(\cdot, \cdot): \mathbb{R} \times \mathbb{R} \rightarrow \mathbb{R}$ such that:

$$
(x, y)=\left\{\begin{array}{ll}
p(y)\left[\frac{x_{1} y_{1}}{p_{0}\left(y_{1}\right)}+\frac{x_{2} y_{2}}{p_{0}\left(y_{2}\right)}\right], & \text { for } y_{1} \neq 0 \text { and } y_{2} \neq 0, \\
p(y) \frac{x_{1} y_{1}}{p_{0}\left(y_{1}\right)}, & \text { for } y_{1} \neq 0 \text { and } y_{2}=0, \\
p(y) \frac{x_{2} y_{2}}{p_{0}\left(y_{2}\right)}, & \text { for } y_{1}=0 \text { and } y_{2} \neq 0, \\
0, & \text { for } y_{1}=0 \text { and } y_{2}=0 .
\end{array} .\right.
$$

The function defined above have the following properties:

1) $(x, x) \geq 0, \forall\left(x_{1}, x_{2}\right) \in \mathbb{R}^{2}$.

2) For $\lambda>0$

$$
(x, \lambda y)=p(\lambda y)\left[\frac{x_{1}\left(\lambda y_{1}\right)}{p_{0}\left(\lambda y_{1}\right)}+\frac{x_{2}\left(\lambda y_{2}\right)}{p_{0}\left(\lambda y_{2}\right)}\right]=\lambda^{2} p(y)\left[\frac{\left.x_{1} y_{1}\right)}{\lambda p_{0}\left(y_{1}\right)}+\frac{x_{2} y_{2}}{\lambda p_{0}\left(y_{2}\right)}\right]
$$




$$
=\lambda p(y)\left[\frac{\left.x_{1} y_{1}\right)}{p_{0}\left(y_{1}\right)}+\frac{x_{2} y_{2}}{p_{0}\left(y_{2}\right)}\right] .
$$

$$
(\lambda x, y)=p(y)\left[\frac{\left(\lambda x_{1}\right) y_{1}}{p_{0}\left(y_{1}\right)}+\frac{\left(\lambda x_{2}\right) y_{2}}{p_{0}\left(y_{2}\right)}\right]=\lambda p(y)\left[\frac{x_{1} y_{1}}{p_{0}\left(y_{1}\right)}+\frac{x_{2} y_{2}}{p_{0}\left(y_{2}\right)}\right]=\lambda(x, y), \lambda \in \mathbb{R} .
$$

3) $\left(x+x^{\prime}, y\right)=(x, y)+\left(x^{\prime}, y\right)$

Case 1. $x=\left(x_{1}, x_{2}\right), x^{\prime}=\left(x_{1}^{\prime}, x_{2}^{\prime}\right)$ and $y=\left(y_{1}, y_{2}\right)$ where $x_{1} \neq 0, x_{2} \neq 0$, $x_{1}^{\prime} \neq 0, x_{2}^{\prime} \neq 0$ :

$$
\begin{aligned}
\left(x+x^{\prime}, y\right) & =p(y)\left[\frac{\left(x_{1}+x_{1}^{\prime}\right) y_{1}}{p_{0}\left(y_{1}\right)}+\frac{\left(x_{2}+x_{2}^{\prime}\right) y_{2}}{p_{0}\left(y_{2}\right)}\right] \\
& =p(y)\left[\frac{x_{1} y_{1}}{p_{0}\left(y_{1}\right)}+\frac{x_{1}^{\prime} y_{1}}{p_{0}\left(y_{1}\right)}+\frac{x_{2} y_{2}}{p_{0}\left(y_{2}\right)}+\frac{x_{2}^{\prime} y_{2}}{p_{0}\left(y_{2}\right)}\right] \\
& =p(y)\left[\frac{x_{1} y_{1}}{p_{0}\left(y_{1}\right)}+\frac{x_{2} y_{2}}{p_{0}\left(y_{2}\right)}\right]+p(y)\left[\frac{x_{1}^{\prime} y_{1}}{p_{0}\left(y_{1}\right)}+\frac{x_{2}^{\prime} y_{2}}{p_{0}\left(y_{2}\right)}\right] \\
& =(x, y)+\left(x^{\prime}, y\right) .
\end{aligned}
$$

Case 2. $x=\left(x_{1}, x_{2}\right), x^{\prime}=\left(x_{1}^{\prime}, 0\right)$ and $y=\left(y_{1}, y_{2}\right)$ where $x_{1} \neq 0, x_{2} \neq 0$, $x_{1}^{\prime} \neq 0$ :

$$
(x, y)=p(y)\left[\frac{x_{1} y_{1}}{p_{0}\left(y_{1}\right)}+\frac{x_{2} y_{2}}{p_{0}\left(y_{2}\right)}\right] \text { and }\left(x^{\prime}, y\right)=p(y) \frac{x_{1}^{\prime} y_{1}}{p_{0}\left(y_{1}\right)}
$$

In this case $x+x^{\prime}=\left(x_{1}+x_{1}^{\prime}, x_{2}\right)$ therefore:

$$
\begin{aligned}
\left(x+x^{\prime}, y\right) & =p(y)\left[\frac{\left(x_{1}+x_{1}^{\prime}\right) y_{1}}{p_{0}\left(y_{1}\right)}+\frac{x_{2} y_{2}}{p_{0}\left(y_{2}\right)}\right] \\
& =p(y)\left[\frac{x_{1} y_{1}}{p_{0}\left(y_{1}\right)}+\frac{x_{2} y_{2}}{p_{0}\left(y_{2}\right)}\right]+p(y) \frac{x_{1}^{\prime} y_{1}}{p_{0}\left(y_{1}\right)} \\
& =(x, y)+\left(x^{\prime}, y\right) .
\end{aligned}
$$

The reconciliation $\left(x+x^{\prime}, y\right)=(x, y)+\left(x^{\prime}, y\right)$ goes equally in these cases:

a) $x=\left(x_{1}, x_{2}\right), x^{\prime}=\left(0, x_{2}^{\prime}\right)$ and $y=\left(y_{1}, y_{2}\right)$ where $x_{1} \neq 0, x_{2} \neq 0, x_{2}^{\prime} \neq 0$

b) $x=\left(x_{1}, 0\right), x^{\prime}=\left(x_{1}^{\prime}, x_{2}^{\prime}\right)$ and $y=\left(y_{1}, y_{2}\right)$ where $x_{1} \neq 0, x_{1}^{\prime} \neq 0, x_{2}^{\prime} \neq 0$

c) $x=\left(0, x_{2}\right), x^{\prime}=\left(x_{1}^{\prime}, x_{2}^{\prime}\right)$ and $y=\left(y_{1}, y_{2}\right)$ where $x_{2} \neq 0, x_{1}^{\prime} \neq 0, x_{2}^{\prime} \neq 0$

Case 3: $x=\left(x_{1}, x_{2}\right), x^{\prime}=\left(x_{1}^{\prime}, x_{2}^{\prime}\right)$ and $y=\left(y_{1}, y_{2}\right)$ where $x_{1} \neq 0, x_{2} \neq 0$, $x_{1}^{\prime} \neq 0, x_{2}^{\prime} \neq 0$ but $x_{1}+x_{1}^{\prime}=0$ and $x_{2}+x_{2}^{\prime}=0$ so $x_{1}=-x_{1}^{\prime}$ and $x_{2}=-x_{2}^{\prime}$.

In this case $x+x^{\prime}=(0,0)$ therefore $\left(x+x^{\prime}, y\right)=0$ while:

$$
\begin{aligned}
\left(x^{\prime}, y\right) & =p(y)\left[\frac{x_{1}^{\prime} y_{1}}{p_{0}\left(y_{1}\right)}+\frac{x_{2}^{\prime} y_{2}}{p_{0}\left(y_{2}\right)}=p(y)\left[\frac{-x_{1} y_{1}}{p_{0}\left(y_{1}\right)}+\frac{-x_{2} y_{2}}{p_{0}\left(y_{2}\right)}\right]\right. \\
& =-p(y)\left[\frac{x_{1} y_{1}}{p_{0}\left(y_{1}\right)}+\frac{x_{2} y_{2}}{p_{0}\left(y_{2}\right)}\right]=-(x, y)
\end{aligned}
$$

from where: $(x, y)+\left(x^{\prime}, y\right)=0=\left(x+x^{\prime}, y\right)$. 
Case 4: $x=\left(x_{1}, x_{2}\right), x^{\prime}=\left(x_{1}^{\prime}, x_{2}^{\prime}\right)$ and $y=\left(y_{1}, y_{2}\right)$ where $x_{1} \neq 0, x_{2} \neq 0$, $x_{1}^{\prime} \neq 0, \quad x_{2}^{\prime} \neq 0$ but $x_{1}+x_{1}^{\prime}=0$ so $x_{1}=-x_{1}^{\prime}$.

In this case $x+x^{\prime}=\left(0, x_{2}+x_{2}^{\prime}\right)$ therefore:

while:

$$
\left(x+x^{\prime}, y\right)=p(y) \frac{\left(x_{2}+x_{2}^{\prime}\right) y_{2}}{p_{0}\left(y_{2}\right)}=p(y) \frac{x_{2} y_{2}}{p_{0}\left(y_{2}\right)}+p(y) \frac{x_{2}^{\prime} y_{2}}{p_{0}\left(y_{2}\right)}
$$

and

$$
(x, y)=p(y)\left[\frac{x_{1} y_{1}}{p_{0}\left(y_{1}\right)}+\frac{x_{2} y_{2}}{p_{0}\left(y_{2}\right)}\right]
$$

$$
\left(x^{\prime}, y\right)=p(y)\left[\frac{x_{1}^{\prime} y_{1}}{p_{0}\left(y_{1}\right)}+\frac{x_{2}^{\prime} y_{2}}{p_{0}\left(y_{2}\right)}\right]=p(y)\left[\frac{-x_{1} y_{1}}{p_{0}\left(y_{1}\right)}+\frac{x_{2}^{\prime} y_{2}}{p_{0}\left(y_{2}\right)}\right]
$$

Since, from $(x, y)+\left(x^{\prime}, y\right)=p(y) \frac{x_{2} y_{2}}{p_{0}\left(y_{2}\right)}+p(y) \frac{x_{2}^{\prime} y_{2}}{p_{0}\left(y_{2}\right)}=\left(x+x^{\prime}, y\right)$.

It is equally demonstrated when: $x=\left(x_{1}, x_{2}\right), x^{\prime}=\left(x_{1}^{\prime}, x_{2}^{\prime}\right)$ and $y=\left(y_{1}, y_{2}\right)$ where $x_{1} \neq 0, x_{2} \neq 0, x_{1}^{\prime} \neq 0, x_{2}^{\prime} \neq 0$ but $x_{2}+x_{2}^{\prime}=0$ so $x_{2}=-x_{2}^{\prime}$.

4) From the definition of the function

$$
p_{0}(x)=\left\{\begin{array}{l}
|x|, \quad x<0 \\
2|x|, x \geq 0
\end{array}\right.
$$

we obtain the inequality: $|x| \leq p_{0}(x), \forall x \in \mathbb{R}^{2}$, from where:

brings:

$$
\left|x_{1}\right| \leq p_{0}\left(x_{1}\right) \wedge\left|x_{2}\right| \leq p_{0}\left(x_{2}\right),\left|y_{1}\right| \leq p_{0}\left(y_{1}\right) \wedge\left|y_{2}\right| \leq p_{0}\left(y_{2}\right)
$$

$$
\begin{aligned}
|(x, y)| & \leq p(y)\left[\left|x_{1}\right| \frac{\left|y_{1}\right|}{p_{0}\left(y_{1}\right)}+\left|x_{2}\right| \frac{\left|y_{2}\right|}{p_{0}\left(y_{2}\right)}\right] \\
& =p(y)\left[\left|x_{1}\right|+\left|x_{2}\right|\right] \leq p(y)\left[p_{0}\left(x_{1}\right)+p_{0}\left(x_{2}\right)\right] \\
& =p(y) p(x)=p(x) p(y) .
\end{aligned}
$$

So $|(x, y)| \leq p(x) p(y)$, from where $(x, x)=|(x, x)| \leq p^{2}(x)$.

Remark. For $(x, x)$ where $x\left(x_{1}, x_{2}\right) \in \mathbb{R}^{2}$ we have:

1) $x_{1} \neq 0, x_{2} \neq 0 \Rightarrow p\left(x_{1}\right) \neq 0, p\left(x_{2}\right) \neq 0 \Rightarrow$

$$
\begin{aligned}
(x, x) & =p(x)\left[\frac{x_{1}^{2}}{p_{0}\left(x_{1}\right)}+\frac{x_{2}^{2}}{p_{0}\left(x_{2}\right)}\right]=p(x)\left[\frac{\left|x_{1}\right|^{2}}{p_{0}\left(x_{1}\right)}+\frac{\left|x_{2}\right|^{2}}{p_{0}\left(x_{2}\right)}\right] \\
& \leq p(x)\left[p_{0}\left(x_{1}\right)+p_{0}\left(x_{2}\right)\right]=p^{2}(x) .
\end{aligned}
$$

2) $x_{1} \neq 0, x_{2}=0 \Rightarrow p\left(x_{1}\right) \neq 0, p\left(x_{2}\right)=0 \Rightarrow$

$$
(x, x)=p(x) \frac{x_{1}^{2}}{p_{0}\left(x_{1}\right)}=\left|x_{1}\right|^{2} \leq p_{0}^{2}(x)=p^{2}(x)
$$

3) $x_{1}=0, x_{2} \neq 0 \Rightarrow p\left(x_{1}\right)=0, p\left(x_{2}\right) \neq 0$ 


$$
(x, x)=p(x) \frac{x_{2}^{2}}{p_{0}\left(x_{2}\right)}=\left|x_{2}\right|^{2} \leq p_{0}^{2}(x)=p^{2}(x)
$$

4) $x_{1}=0, x_{2}=0 \Rightarrow p\left(x_{1}\right)=p\left(x_{2}\right)=0 \Rightarrow p(x)=0 \Rightarrow(x, x)=0=p^{2}(x)$

Finally: $|(x, x)| \leq p^{2}(x)$.

Remark. Frankly, $(x, x)=p^{2}(x)$ every time is not true. Because for $x=(-1,2)$ we have $p(x)=|-1|+2|2|=5 \Rightarrow p^{2}(x)=25$ and other side:

$$
(x, x)=p(x)\left[\frac{x_{1}^{2}}{p_{0}\left(x_{1}\right)}+\frac{x_{2}^{2}}{p_{0}\left(x_{2}\right)}\right]=5\left[\frac{(-1)^{2}}{|-1|}+\frac{2^{2}}{2|2|}\right]=5[1+1]=10 .
$$

In this case $(x, x) \neq p^{2}(x)$.

Record 1. Also we can prove that $p^{2}(x) \leq 2(x, x)$.

Proof. Case 1: For $x\left(x_{1}, x_{2}\right) \in \mathbb{R}^{2}$ where $x_{1}<0 \wedge x_{2}<0$ we have:

$$
(x, x)=p(x)\left[\frac{x_{1}^{2}}{p_{0}\left(x_{1}\right)}+\frac{x_{2}^{2}}{p_{0}\left(x_{2}\right)}\right]=p(x)\left[\frac{\left|x_{1}\right|^{2}}{\left|x_{1}\right|}+\frac{\left|x_{2}\right|^{2}}{\left|x_{2}\right|}\right]=p(x)\left[\left|x_{1}\right|+\left|x_{2}\right|\right]=p(x) p(x)
$$

or $p^{2}(x)=(x, x) \leq 2(x, x)$

Case 2: For $x\left(x_{1}, x_{2}\right) \in \mathbb{R}^{2}$ where $x_{1}>0 \wedge x_{2}>0$ we have:

$$
(x, x)=p(x)\left[\frac{x_{1}^{2}}{p_{0}\left(x_{1}\right)}+\frac{x_{2}^{2}}{p_{0}\left(x_{2}\right)}\right]=p(x)\left[\frac{\left|x_{1}\right|^{2}}{2\left|x_{1}\right|}+\frac{\left|x_{2}\right|^{2}}{2\left|x_{2}\right|}\right]=p(x)\left[\frac{\left|x_{1}\right|}{2}+\frac{\left|x_{2}\right|}{2}\right]=\frac{p^{2}(x)}{2} .
$$

So $p^{2}(x)=2(x, x)$.

Case 3: For $x\left(x_{1}, x_{2}\right) \in \mathbb{R}^{2}$ and $x_{1}>0 \wedge x_{2}<0\left[x_{1}<0 \wedge x_{2}>0\right]$ we have:

$$
\begin{aligned}
(x, x) & =p(x)\left[\frac{x_{1}^{2}}{p_{0}\left(x_{1}\right)}+\frac{x_{2}^{2}}{p_{0}\left(x_{2}\right)}\right]=p(x)\left[\frac{\left|x_{1}\right|^{2}}{2\left|x_{1}\right|}+\frac{\left|x_{2}\right|^{2}}{\left|x_{2}\right|}\right] \\
& =p(x)\left[\frac{\left|x_{1}\right|}{2}+\left|x_{2}\right|\right] \geq p(x)\left[\frac{\left|x_{1}\right|}{2}+\frac{\left|x_{2}\right|}{2}\right]=\frac{p^{2}(x)}{2} .
\end{aligned}
$$

So $p^{2}(x) \leq 2(x, x)$.

Record 2. The function $(x, y)$ defined as above provides the benefit of the function $\bar{p}: \mathbb{R} \times \mathbb{R} \rightarrow \mathbb{R}$ such that: $\bar{p}(x)=\sqrt{(x, x)}$.

From the inequality: $p^{2}(x) \leq 2(x, x)$ we have

$$
p^{2}(x) \leq 2 \bar{p}^{2}(x) \text { or } p(x) \leq \sqrt{2} \bar{p}(x),
$$

and from the inequality $|(x, y)| \leq p(x) p(y)$ we have:

$$
|(x, y)| \leq p(x) p(y) \leq \sqrt{2} \bar{p}(x) \sqrt{2} \bar{p}(y)=2 \bar{p}(x) \bar{p}(y) .
$$

So for the function $\bar{p}: \mathbb{R} \times \mathbb{R} \rightarrow \mathbb{R}$ these properties hold: 
1) $\bar{p}(x) \geq 0, \bar{p}(x)=0 \Rightarrow x=0$ for $x \in \mathbb{R}^{2}$

2) $\bar{p}(\lambda x)=\lambda \bar{p}(x)$, for $\lambda>0, x \in \mathbb{R}^{2}$

3) for $x, y \in \mathbb{R}^{2}$ :

$$
\begin{aligned}
\bar{p}^{2}(x+y) & =|(x+y, x+y)| \leq|(x, x+y)|+|(y, x+y)| \\
& \leq 2 \bar{p}(x) \bar{p}(x+y)+2 \bar{p}(y) \bar{p}(x+y) \\
& =2 \bar{p}(x+y)[\bar{p}(x)+\bar{p}(y)]
\end{aligned}
$$

So $\bar{p}(x+y) \leq 2[\bar{p}(x)+\bar{p}(y)]$, for $x, y \in \mathbb{R}^{2}$.

\section{MAin ReSUlts}

Definition 2. The function $(\cdot, \cdot): X \times X \rightarrow \mathbb{R}$, where $X$ is a vectorial space, it is called the asymmetric quasi inner product if:
a) $(x, x) \geq 0, \forall x \in X$
b) $(\lambda x, y)=\lambda(x, y), \forall(x, y) \in X^{2}$ and $\forall \lambda \in \mathbb{R}$
$(x, \lambda y)=\lambda(x, y), \forall(x, y) \in X^{2}$ and $\lambda>0$
c) $\left(x+x^{\prime}, y\right)=(x, y)+\left(x^{\prime}+y\right), \forall x, x^{\prime}, y \in X$
d) $|(x, y)|^{2} \leq k(x, x)(y, y)$, for $k \geq 1$.

Definition 3. The function $p: X \rightarrow \mathbb{R}^{+}$it is called the asymmetric quasi norm function if:
a) $p(x) \geq 0, \forall x \in X$
b) $p(\lambda x)=\lambda p(x), \forall x \in X$ and $\lambda>0$
c) $p(x+y) \leq k[p(x)+p(y)], \forall(x, y) \in X^{2}$ and $k \geq 1$.

Proposition 2. If $(x, y)$ is the asymmetric quasi inner product function on $X$, then the function $\bar{p}: X \rightarrow \mathbb{R}$ such that $\bar{p}(x)=\sqrt{(x, x)}$ is an asymmetric quasi norm function.

Proof. 1) We have

$$
\bar{p}(x)=\sqrt{(x, x)} \geq 0, \forall x \in X
$$

2) We have

$$
\bar{p}(\lambda x)=\sqrt{(\lambda x, \lambda x)}=\sqrt{\lambda^{2}(x, x)} \text {, for } \lambda>0 \text {. }
$$

Therefore

$$
\bar{p}(\lambda x)=|\lambda| \sqrt{(x, x)}=\lambda \bar{p}(x) .
$$


3) We have

$$
\begin{aligned}
\bar{p}^{2}(x+y) & =|(x+y, x+y)| \\
& =|(x, x+y)+(y, x+y)| \\
& \leq|(x, x+y)|+|(y, x+y)| \\
& \leq \sqrt{k^{\prime}(x, x)(x+y, x+y)}+\sqrt{k^{\prime}(y, y)(x+y, x+y)} \\
& =\sqrt{k^{\prime}} \bar{p}(x) \bar{p}(x+y)+\sqrt{k^{\prime}}-\bar{p}(y) \bar{p}(x+y) \\
& =\sqrt{k^{\prime}}[\bar{p}(x)+\bar{p}(y)] \bar{p}(x+y) .
\end{aligned}
$$

From where: $\bar{p}(x+y) \leq \sqrt{k^{\prime}}[\bar{p}(x)+\bar{p}(y)]$ and if we denote $\sqrt{k^{\prime}}=k \geq 1$ we have:

$$
\bar{p}(x+y) \leq k[\bar{p}(x)+\bar{p}(y)] .
$$

\section{Conclusions}

An asymmetric quasi norm function can be obtained by an asymmetric inner product function, and the link between them is the function: $\bar{p}: X \rightarrow \mathbb{R}$, so that $\bar{p}(x)=\sqrt{(x, x)}$

\section{References}

[1] G. Lumer. Semi- inner products spaces.Trans. Am. Math.Soc. 100 (1961), $29-43$

[2] R. Giles.Classes of semi inner product spaces. Trans.Am. Math.Soc.129 (1967),436-446

[3] L. Tashim. On denseness on asymetric Metric Spaces. Mathematics Aeterna, Vol.2.2012

[4] S. Cobzas. Functional on Asymmetric Normed Spaces. Springer. Fronties in Mathematics, 2013. ISBN.978-3-0348-0478-3

${ }^{1)}$ Faculty of Natural Sciences, Department of Mathematics, "Aleksandër Xhuvani” University, Elbasan, Albania E-mail address: stelaceno85@yahoo.com

${ }^{2)}$ Faculty of Natural Sciences, Department of Mathematics, "Aleksandër Xhuvani" University, Elbasan, Albania E-mail address: gladi.tigno@yahoo.com 